\title{
Thermal Stability of Nanostructured Synthetic Ferrimagnets under Applied Magnetic Fields in the $\mathbf{4 5}^{\circ}$ Direction
}

\author{
C. W. Han, J. K. Han, and S. H. Lim* \\ Department of Materials Science and Engineering, Korea University, Seoul 136-713, Korea
}

(Received 3 August 2010, Received in final form 9 September 2010, Accepted 9 September 2010)

\begin{abstract}
An accurate analytical equation for the total energy in the framework of the single domain model is used to study the thermal stability of nanostructured synthetic ferrimagnets. Elliptical cells are considered that have lateral dimensions of $160 \mathrm{~nm}$ (long axis) $\times 80 \mathrm{~nm}$ (short axis) and varying values of thickness asymmetry for the two magnetic layers. The direction of the applied magnetic field, which points to the $45^{\circ}$ direction, is in the opposite direction to the thicker layer magnetization. A significant difference is observed in the applied magnetic field dependencies of the equilibrium magnetic configuration and the magnetic energy barrier when using the simplifying assumption that the self-demagnetizing field is identical in magnitude to the dipole field. At a small thickness asymmetry of $0.2 \mathrm{~nm}$, for example, the magnetic energy barrier is reduced from $68 \mathrm{kT}(T=300$ K) to $6 \mathrm{kT}$ at the remanent state and a progressive switching behavior changes into a critical behavior, as the simplifying assumption is used. The present results clearly demonstrate the need for an accurate analytical equation for the total energy in predicting the thermal stability of nanostructured synthetic ferrimagnets.
\end{abstract}

Keywords : synthetic ferrimagnets, nanostructured cells, magnetic configuration, magnetic energy barrier, analytical equation for the total energy

\section{Introduction}

Because device miniaturization currently progresses at a rapid pace, thermal stability is of particular concern. Nanostructured cells of synthetic ferrimagnets, which are composed of two magnetic layers separated by a very thin nonmagnetic spacer, are being increasingly used in advanced magnetic devices. Recently, Worledge studied the thermal stability of nanostructured synthetic ferrimagnets by using a total energy equation [1]. Either a minimal or no shape anisotropy effect was observed. This is somewhat unexpected for the nanoscale size range explored for this study [2,3]. A simplifying assumption used in the derivation of the equation for the total energy $(E)$ is responsible for this unexpected result, whereby the selfdemagnetizing field $\left(H_{\mathrm{dem}}\right)$ of one layer is equal in magnitude to the dipole (or stray) field $\left(H_{\mathrm{dip}}\right)$ from the other layer if the two magnetic layers have an identical thickness (no thickness asymmetry) and saturation magnetization. Note that at the remanent state where the two magnetizations are aligned antiparallel, the direction of

*Corresponding author: Tel: +82-2-3290-3285

Fax: +82-2-928-3584, e-mail: sangholim@korea.ac.kr
$H_{\text {dem }}$ is opposite to that of $H_{\text {dip. }}$ Recently, the authors were able to propose a more accurate analytical expression for $E$ by removing the simplifying assumption $[4,5]$. The key to this expression is the use of the effective magnetostatic fields (both $H_{\text {dem }}$ and $H_{\text {dip }}$ ), the details of which will be discussed in the next section. The accurate equation for $E$ was previously used to investigate the evolution of the equilibrium magnetic configuration and the magnetic energy barrier $\left(E_{\mathrm{M}}\right)$, which is a direct measure of the thermal stability, under the applied magnetic field $(H)$ in the longitudinal direction [5]. It was found from the previous study [5] that there is a significant difference in the results as the simplifying assumption is removed. Similar work is performed in this present study, with the exception that the direction of $H$ now points to the $45^{\circ}$ direction. This direction of $H$ is relevant to MRAM based on the asteroidswitching when the applied current is switched on both in the word and bit lines. A particular emphasis is placed on the thermal stability of the nanostructured cells.

\section{Model and Calculation}

The synthetic ferrimagnets are comprised of two ferro- 
magnetic layers sandwiching a nonmagnetic spacer such as Ru. Figs. 1(a) and (b) show the top and cross-sectional views, respectively, of the multilayer. In Fig. 1(a), the definition of the $x$ and $y$ axes is also shown, together with the direction of $H$, which is in the $45^{\circ}$ direction. Elliptical cells with lateral dimensions of $160 \mathrm{~nm}$ (long axis) $\times 80$ $\mathrm{nm}$ (short axis) were considered. While the total thickness of the two magnetic layers $\left(t_{1}+t_{2}\right)$ was fixed at $4 \mathrm{~nm}$, the thickness of each magnetic layer was widely varied in order to observe the effects of thickness asymmetry. Here, $t_{1}$ and $t_{2}$ denote the thicknesses of the upper and lower magnetic layers, respectively, and in this study, $t_{1} \geq t_{2}$. The symbol $\Delta t$, defined as $t_{1}-t_{2}$, was used to indicate the thickness asymmetry. With $t_{1} \geq t_{2}$, the direction of $H$ is in the opposite direction to the thicker layer (see Figs. 1(a) and (b)). The thickness of the spacer $\left(t_{\mathrm{s}}\right)$ was fixed at 0.6 $\mathrm{nm}$. The saturation magnetization $\left(M_{\mathrm{s}}\right)$ of the two magnetic layers was $1034 \mathrm{emu} / \mathrm{cc}$, which is relevant to a CoFe-B alloy [6]. The induced anisotropy $\left(H_{\mathrm{i}}\right)$, with a magnitude of $10 \mathrm{Oe}$, was formed in the $x$ direction so that it can be additively combined with the shape anisotropy to give the total anisotropy. The interlayer exchange coupling constant between the two magnetic layers $(J)$ was $-0.17 \mathrm{erg} / \mathrm{cm}^{2}$ (the negative sign indicates the antiferromagnetic coupling). Two simplifying assumptions are used to derive the equation for $E$; the magnetizations in both layers are in the single domain state and they lay only in the film plane. These two assumptions were also used previously by Worledge [1,7]. It is worth mentioning here that, in a very recent study by the present authors [8], the two simplifying assumptions of the single and inplane domain state can also be relaxed, thus resulting in more accurate results. However, since the difference is rather small for the elliptical cell of current interest [8], the two simplifying assumptions are used just for simplicity. The equation for $E$ is expressed as:

$$
\begin{aligned}
E\left(\theta_{1}, \theta_{2}\right)= & -M_{s}\left[H_{a, x}\left(V_{1} \cos \theta_{1}+V_{2} \cos \theta_{2}\right)\right. \\
& \left.+H_{a, y}\left(V_{1} \cos \theta_{1}+V_{2} \cos \theta_{2}\right)\right] \\
& +\frac{1}{2} M_{s}\left[H_{d e m, x-1} V_{1} \cos ^{2} \theta_{1}+H_{d e m, x-2} V_{2} \cos ^{2} \theta_{2}\right. \\
& \left.+H_{d e m, y-1} V_{1} \sin ^{2} \theta_{1}+H_{d e m, y-2} V_{2} \sin ^{2} \theta_{2}\right] \\
& +\frac{1}{2} M_{s}\left[\left(V_{1} H_{d i p, x-2}+V_{2} H_{d i p, x-1}\right) \cos \theta_{1} \cos \theta_{2}\right. \\
& \left.\left(V_{1} H_{d i p, y-2}+V_{2} H_{d i p, y-1}\right) \sin \theta_{1} \sin \theta_{2}\right] \\
& -\frac{1}{2} M_{s} H_{i}\left(V_{1} \cos ^{2} \theta_{1}+V_{2} \cos ^{2} \theta_{2}\right)-A J \cos \left(\theta_{1}-\theta_{2}\right)
\end{aligned}
$$

Here, the subscripts 1 and 2 indicate the magnetic layers 1

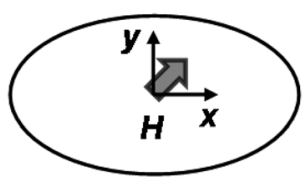

(a)

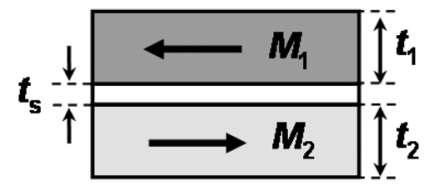

(b)
Fig. 1. (a) Top and (b) cross-sectional views of the synthetic ferrimagnets used in this study. The definition of the axes and the direction of $H$, which is in the opposite direction to the thicker layer (layer 1) magnetization, are also shown in (a). Elliptical cells have lateral dimensions of $160 \mathrm{~nm}$ (long axis) $\times$ $80 \mathrm{~nm}$ (short axis). Note that for clarity the dimensions are not to scale.

and 2, respectively, while the subscripts $x$ and $y$ respectively denote the longitudinal and transverse directions in the film plane (Fig. 1(a)). $V$ is the volume of the magnetic layer, $\theta$ is the angle of the magnetization with respect to the $+x$ axis, and $A$ is the (lateral) area. $H_{\text {dem-x }}$ and $H_{\text {dem-y }}$ are the effective self-demagnetizing fields along the $x$ and $y$ axes respectively. Similar symbols, $H_{\text {dip-x }}$ and $H_{\text {dip-y, }}$, were used for the effective stray fields. The first three terms in Eqn. (1) indicate the Zeeman energies due to $H$, $H_{\text {dem }}$, and $H_{\text {dip }}$, respectively. The last two terms arise from the induced anisotropy energy and the interlayer exchange energy, respectively. Eqn. (1) converges into the energy equation originally used by Worledge when $H_{\mathrm{dem}, \mathrm{x}}=H_{\mathrm{dip}, \mathrm{x}}$ and $H_{\text {dem,y }}=H_{\text {dip,y }}[1,7]$.

The use of the effective fields in Eqn. (1) is necessary for the analytical expression, because the magnetostatic fields are somewhat non-uniform. The effective fields were obtained by averaging the non-uniform fields over the entire magnetic volume. Three dimensional (3D) numerical calculations based on the finite element method (FEM) were performed using a commercial program package (multiphysics finite element method COMSOL) in order to calculate the two magnetostatic fields. The numerical program is able to accurately cope with curved geometry (such as the elliptical cells used here) without the need to be approximated by the cells of a square grid. A sufficiently large number of meshes $(20,000 \sim 30,000)$ were used in order to ensure the accuracy of the calculated results.

Since the accurate analytical expression for $E$ is now available, it is a straightforward task to examine the equilibrium magnetic configuration and $E_{\mathrm{M}}$ (and hence the thermal stability) as a function of $H$. With two angular parameters $\left(\theta_{1}\right.$ and $\left.\theta_{2}\right)$ in the equation, the energy is represented by a surface. The minima of the energy surfaces correspond to the magnetic equilibrium (or metastable) configuration. The $H$ dependence of the magnetic configuration can be obtained by tracing the location of the 
minima as $H$ varies. On the other hand, the value of $E_{\mathrm{M}}$ can be obtained by tracing the lowest energy path linking two energy minima, one of which corresponds to the initial state and the other to the final state. This is because $E_{\mathrm{M}}$ is the difference between the energies at the initial state and at the saddle point. Consequently, it would be preferable for the energy profile to be between the initial and final states along the lowest energy path, because the energy profile can be represented by a more convenient curve (not a surface) and, more importantly, the magnitude of $E_{\mathrm{M}}$ can be obtained directly from the energy profile. Therefore, all the results in this study are represented in terms of the energy profile along the lowest energy path, not by the energy surface.

\section{Results and Discussion}

Fig. 2(a) shows the results for the energy profile at various values of $H$ for the cell with $\Delta t=0$ (no thickness asymmetry). In Fig. 2(a) (as well as in the figures to be shown later), the energy profile is plotted as a function only of $\theta_{1}$. The energy profile as a function of $\theta_{2}$ can be obtained in a straightforward manner by using Eqn. (1). Here, we used the notation $\left(\theta_{1}, \theta_{2}\right)$ to describe the magnetic configuration, whereby its meaning is somewhat obvious (the first angle in the parenthesis indicates $\theta_{1}$ in layer 1 and the second angle denotes $\theta_{2}$ in layer 2). Assume that, at $H=0$, the initial magnetic configuration is $\left(180^{\circ}, 0^{\circ}\right)$ (the same as that shown in Fig. 1(b)). No critical behavior is observed as the equilibrium magnetic configuration varies continuously with the increase of $H$. In the small $H$ range ( $H<\sim 300$ Oe), the magnetic configuration is similar to the initial antiparallel alignment. However, as $H$ increases further, the deviation from the antiparallel alignment occurs and the configuration then transforms into a scissoring state with the antiparallel axis in the $45^{\circ}$ direction. Saturation finally occurs at a large value of $H(\sim 2500 \mathrm{Oe})$. The value of $E_{\mathrm{M}}$ (as shown in the inset in Fig. 2(a)), remains nearly constant at $69 \mathrm{kT}$ ( $T$ is assumed to be $300 \mathrm{~K}$ in this study) in the low $H$ range up to 300 Oe and then increases with the increase of $H$. At $H=1010 \mathrm{Oe}$, the value of $E_{\mathrm{M}}$ reaches a maximum, followed by a monotonic decrease with the further increase of $H$. Finally, the value of $E_{\mathrm{M}}$ reaches zero at $H=2040 \mathrm{Oe}$, where saturation of the two magnetizations occurs in the $H$ direction.

Similarly to Fig. 2(a), the results for the energy profile obtained under the simplifying assumption, namely, $H_{\mathrm{dem}, \mathrm{x}}$ $=H_{\mathrm{dip}, \mathrm{x}}$ and $H_{\mathrm{dem}, \mathrm{y}}=H_{\mathrm{dip}, \mathrm{y}}$, are shown in Fig. 2(b). A comparison of the two sets of results shown in Figs. 2(a) and (b) appears to indicate a somewhat similar behavior. How-
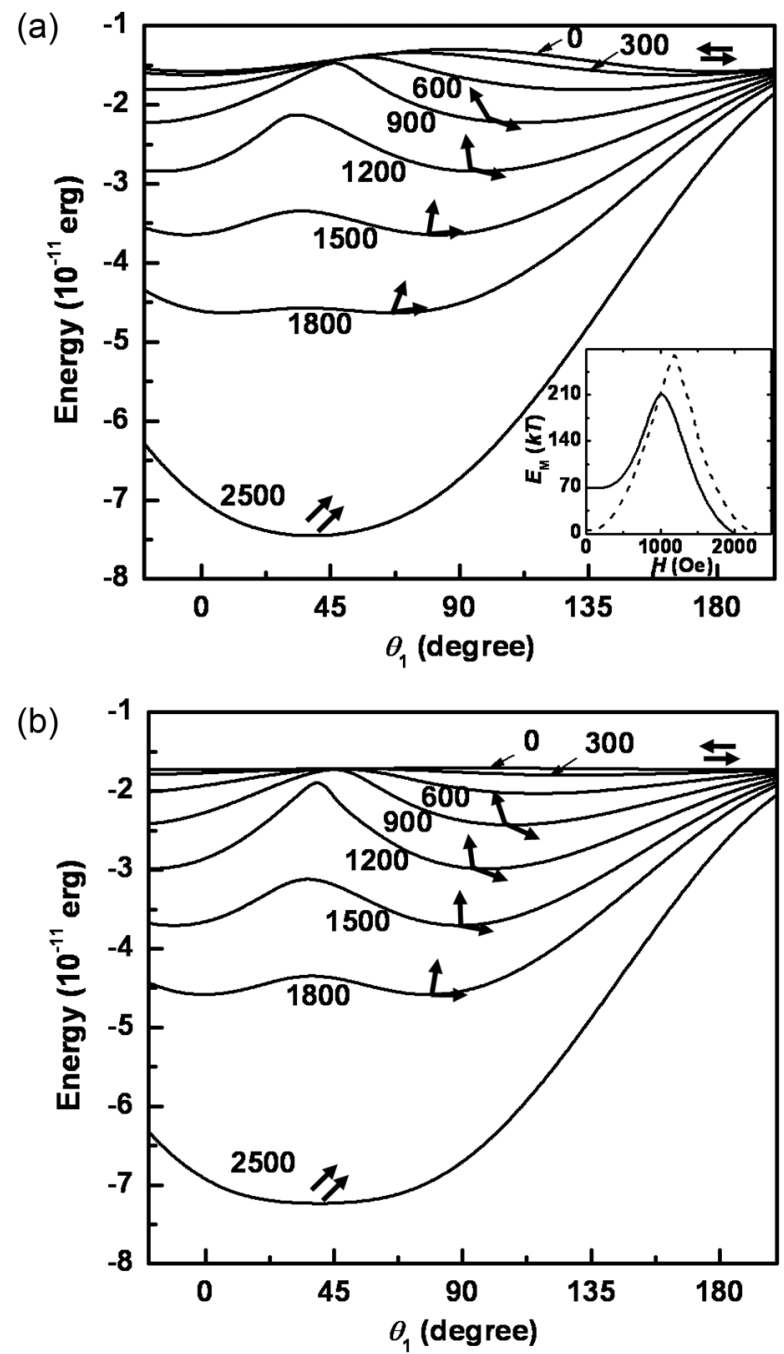

Fig. 2. (a) Results for the angular dependence of the lowest energy path between initial and final states at various values of $H$ for the cell with $\Delta t=0$ (no thickness asymmetry). (b) A similar set of results for the cell with $\Delta t=0$, obtained under the simplifying assumption that $H_{\mathrm{dem}, \mathrm{x}}=H_{\mathrm{dip}, \mathrm{x}}$ and $H_{\mathrm{dem}, \mathrm{y}}=H_{\mathrm{dip}, \mathrm{y}}$. The inset in (a) shows the results for $E_{\mathrm{M}}$ (in $k T$ with $T=300 \mathrm{~K}$ ) as a function of $H$ obtained without the simplifying assumption (solid line) and with the simplifying assumption (dotted line). The initial magnetic configuration is $\left(180^{\circ}, 0^{\circ}\right)$ and the numbers at the curves indicate the magnitude of $H$ in Oe. The arrows indicate the equilibrium magnetic configuration at respective $H$ values.

ever, a closer comparison indicates a substantial difference between the two sets of results. A particular difference is observed in $E_{\mathrm{M}}$ (the results of which are shown in the inset of Fig. 2(a) which shows a clearer comparison with the results obtained) without using the simplifying assumption. At $H=0$ (remanent state), the value of $E_{\mathrm{M}}$ is very small, at only $5 \mathrm{kT}$, which can be compared with the value of $69 \mathrm{kT}$ obtained without using the simplifying 
assumption. This small value of $E_{\mathrm{M}}$ under the simplifying assumption can actually be expected, because, at $\Delta t=0$, $H_{\text {dem }}$ is completely cancelled out by $H_{\text {dip }}$ in both the $x$ and $y$ directions, resulting in zero shape anisotropy. In this case, the intrinsic induced anisotropy $\left(H_{\mathrm{i}}=10\right.$ Oe in this study) only contributes to $E_{\mathrm{M}}$. In reality, however, a complete cancellation does not occur between $H_{\text {dem }}$ and $H_{\text {dip }}$, although the spacer layer is very thin $(0.6 \mathrm{~nm})$. Specifically, at $\Delta t=0$, the following values for the magnetostatic fields are obtained from the present FEM calculations; $H_{\text {dem }, \mathrm{x}}=208 \mathrm{Oe}, H_{\mathrm{dip}, \mathrm{x}}=139 \mathrm{Oe}, H_{\mathrm{dem}, \mathrm{y}}=552 \mathrm{Oe}$, and $H_{\mathrm{dip}, \mathrm{y}}$ $=355$ Oe. These results for the magnetostatic fields lead to a large shape anisotropy of 128 Oe, which is significantly higher than the value of 10 Oe for $H_{\mathrm{i}}$. The small value of $E_{\mathrm{M}}(5 \mathrm{kT})$ is maintained up to $150 \mathrm{Oe}$, above which it increases significantly with the increase of $H$, reaching a peak value (269 kT) at $H=1188 \mathrm{Oe}$, followed by a decrease with the further increase of $H$. The degree of the increment and the decrement after the peak and also the peak value itself are higher than those obtained without the assumption. The value of $E_{\mathrm{M}}$ reaches zero at $H=2310 \mathrm{Oe}$, where saturation of the two magnetizations occurs in the $H$ direction.

It is important to note that, from the inset of Fig. 2(a), at $H>990$ Oe the magnitude of $E_{\mathrm{M}}$ is larger indicating stronger shape anisotropy with the use of the simplifying assumption, which is in striking contrast with the behavior observed in the low $H$ range. This can be explained as follows. In the low $H$ range where the magnetizations are antiparallel (or close to this alignment), the shape anisotropy is zero (or very small) due to the complete (or partial) cancellation of $H_{\mathrm{dem}}$ by $H_{\mathrm{dip}}$, as has been previously mentioned. Note that, in this case, the direction of $H_{\mathrm{dem}}$ is opposite to that of $H_{\text {dip. }}$. However, in the high $H$ range where the magnetizations are in a scissoring state or even saturation, $H_{\text {dem }}$ and $H_{\text {dip }}$ couple constructively causing large shape anisotropy, because the direction of $H_{\mathrm{dem}}$ is the same (or parallel at saturation) as that of $H_{\text {dip. }}$. The higher saturation field observed under the simplifying assumption ( 2405 Oe vs. 2138 Oe) can also be similarly explained. It is important to point out the large discrepancy between the two sets of results for $E_{\mathrm{M}}$ as shown in the inset of Fig. 2(a), the discrepancy being particularly significant in the low $H$ range which is more relevant to real applications. The value of $E_{\mathrm{M}}$ at the remanent state, for example, is predicted to be only $5 \mathrm{kT}$ under the simplifying assumption, indicating that the cell is thermally unstable. This prediction is actually incorrect; the value of $E_{\mathrm{M}}(69 \mathrm{kT})$ after removing the simplifying assumption is high enough for high density MRAM applications [4]. These results clearly demonstrate the importance of using
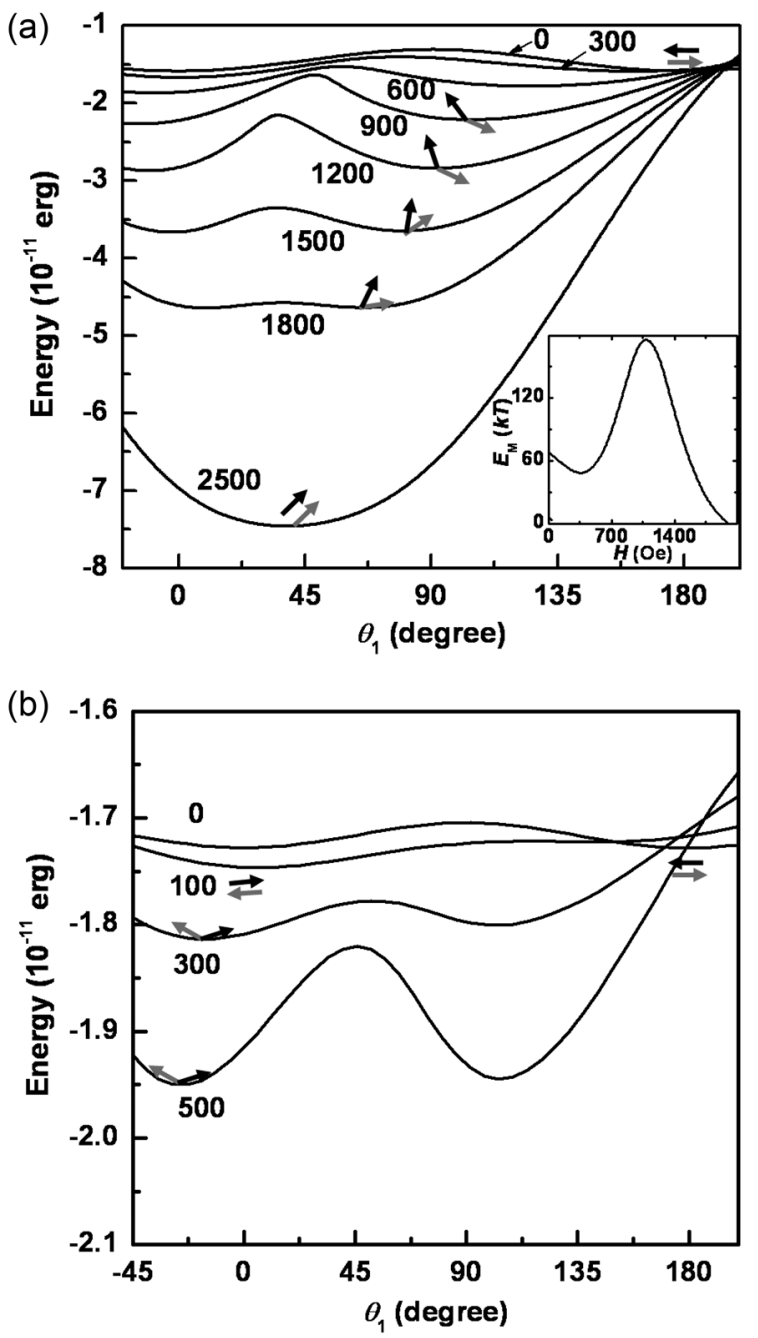

Fig. 3. Results for the angular dependence of the lowest energy path between initial and final states at various values of $H$ for the cell with $\Delta t=0.2 \mathrm{~nm}$. The results are shown similarly to those shown in Figs. 2(a) and (b), with the exception of the different colored arrows; the black arrow indicates the magnetization direction of layer 1 (thicker layer), while the gray arrow denotes the magnetization direction of layer 2 (thinner layer). The results for $E_{\mathrm{M}}$ (in $\mathrm{kT}$ with $T=300 \mathrm{~K}$ ) as a function of $H$ obtained by using the simplifying assumption are also shown in the inset of Fig. 3(a), but they are not clearly shown in the figure, because the magnitude is very small $(6 \mathrm{kT})$ and it decreases to zero at a small field of 100 Oe.

the correct model.

The results of the $H$ dependencies of the equilibrium magnetic configuration and $E_{\mathrm{M}}$ in the cell that has a small thickness asymmetry of $\Delta t=0.2 \mathrm{~nm}$, as shown in Fig. 3(a), are qualitatively similar to those shown in Fig. 2(a) for the symmetric cell. Specifically, the change in the magnetic configuration is continuous and progressive with the increase of $H$. A slight difference is that the change in the 
(a)

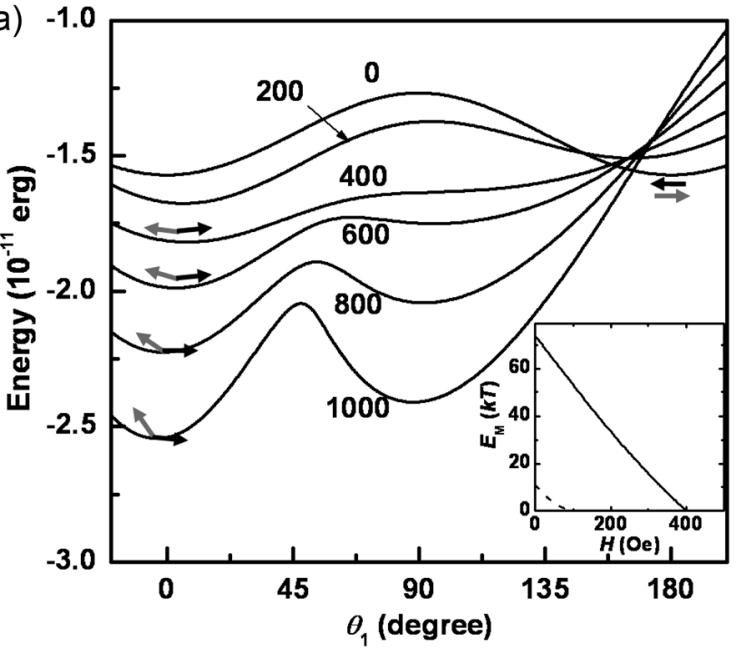

(b)

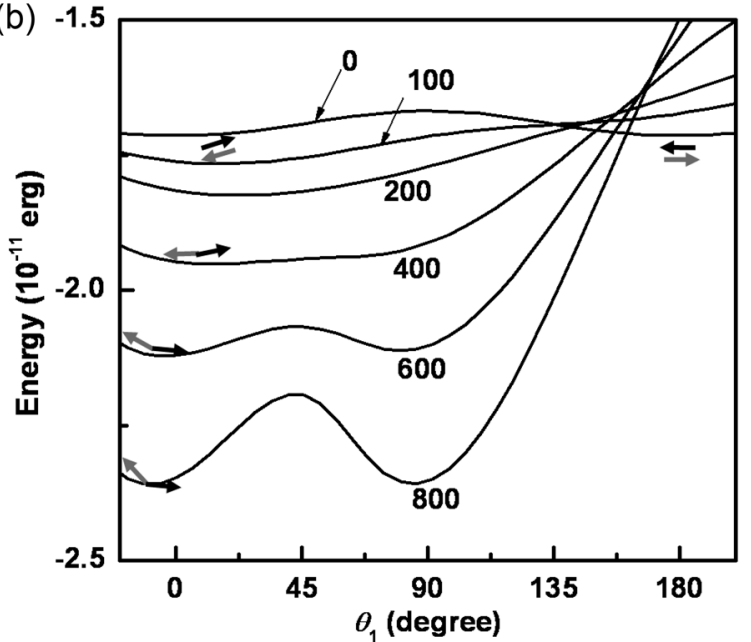

Fig. 4. Results for the angular dependence of the lowest energy path between initial and final states at various values of $H$ for the cell with $\Delta t=0.6 \mathrm{~nm}$. The results are shown similarly to those shown in Figs. 3(a) and 3(b).

magnetic configuration in this asymmetric cell occurs more rapidly as $H$ increases in the low field range, although this is not clear from the results shown in Fig. 3(a) due to the large step of $H$ and also the large scale of the $y$ axis. This tendency increases with an increase of $\Delta t$, due to an increase of the Zeeman energy with the increase of $\Delta t$. Another difference that should be noted is the variation of $E_{\mathrm{M}}$ with $H$, as can be seen more clearly in Fig. 5, where the results for $E_{\mathrm{M}}$ are shown as a function of $H$ for all the values of $\Delta t$ investigated in this study. Although a more detailed discussion is to be made in a later section, the value of $E_{\mathrm{M}}$ initially decreases, reaching a minimum at $H=360$ Oe. This behavior is in contrast to that observed in the cell with $\Delta t=0$, where $E_{\mathrm{M}}$ remains nearly constant up to $300 \mathrm{Oe}$. In the intermediate and high $H$ range, the $H$ dependence of $E_{\mathrm{M}}$ is similar to that observed in the symmetric cell.

Again, a significant difference is observed in the $H$ dependences of the equilibrium magnetic configuration and $E_{\mathrm{M}}$ when the results are calculated under the simplifying assumption, as can be seen from the results shown in Fig. 3(b). The most prominent difference is that magnetization switching occurs in a critical manner through the direct write mode at an $H$ value as small as 100 Oe. Small shape anisotropy due to the cancellation of $H_{\text {dem }}$ by $H_{\text {dip }}$ is responsible for this critical switching behavior. Above this field, the magnetizations change into a scissoring state with the antiparallel axis in the $45^{\circ}$ direction. As can be expected from the large difference in the magnetic configuration, an equally large difference is also observed in the variation of $E_{\mathrm{M}}$ with $H$, as can be seen in the inset of Fig. 3(a). The value of $E_{\mathrm{M}}$ is again very small (6 kT) at the remanent state $(H=0)$ and it quickly reduces to zero at $H=100$ Oe where the switching occurs in the direct write mode.

The results for the energy profile for the cell with a relatively large $\Delta t$ of $0.6 \mathrm{~nm}$ are shown in Figs. 4(a) and (b), similarly to those shown in Figs. 3(a) and (b) for a cell with $\Delta t=0.2 \mathrm{~nm}$. In both cases (with and without the simplifying assumption), the switching occurs in the direct write mode and this is due to large Zeeman effects at this large $\Delta t$. A similar behavior is observed for the cells with $\Delta t \geq 0.8 \mathrm{~nm}$. After the direct write switching, the change in the magnetic configuration is continuous and progressive, similarly to the behavior seen at smaller $\Delta t$ values; namely, the spin scissoring state occurs, followed by the saturation in the $H$ direction.

The results for $E_{\mathrm{M}}$ are summarized in Fig. 5 as a

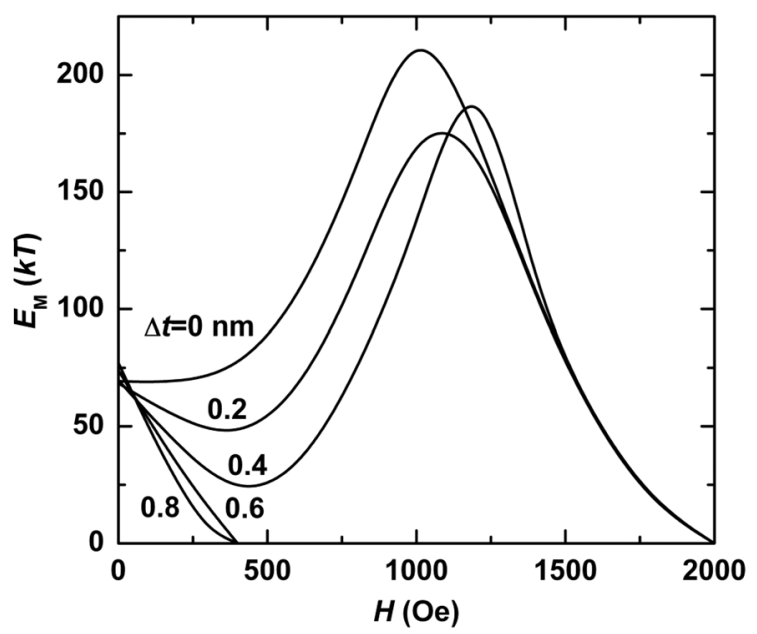

Fig. 5. Results for $E_{\mathrm{M}}$ (in $\mathrm{kT}$ with $T=300 \mathrm{~K}$ ) as a function of $H$ for the cells with $\Delta t=0,0.2 \mathrm{~nm}, 0.4 \mathrm{~nm}, 0.6 \mathrm{~nm}$, and 0.8 $\mathrm{nm}$. 
function of $H$ for all the values of $\Delta t$ investigated in this study in order to illustrate a clearer comparison of the results depending on $\Delta t$. At the remanent state $(H=0)$, the value of $E_{\mathrm{M}}$ increases appreciably from $68 \mathrm{kT}$ to $77 \mathrm{kT}$ as $\Delta t$ increases from 0 to $0.8 \mathrm{~nm}$, indicating that the cancellation of the two magnetostatic fields decreases with the increase of $\Delta t$. For the cells with nonzero $\Delta t, E_{\mathrm{M}}$ decreases initially with the increase of $H$, in contrast to the behavior seen at $\Delta t=0$, where $E_{\mathrm{M}}$ remains nearly constant. Furthermore, in this low $H$ range, the decrease of $E_{\mathrm{M}}$ is steeper at larger $\Delta t$ values due to stronger Zeeman effects. Consequently, the value of $E_{\mathrm{M}}$ with respect to $\Delta t$ is completely reversed ( $E_{\mathrm{M}}$ is lowest at $\Delta t=0.8 \mathrm{~nm}$, while it is highest at $\Delta t=0)$ at $H$ values as small as 55 Oe. At $\Delta t=0.2$ and $0.4 \mathrm{~nm}$, a minimum occurs at intermediate $H$ values. On the other hand, no minimum is observed at large thickness asymmetries of $\Delta t \geq 0.6 \mathrm{~nm}$ because the value of $E_{\mathrm{M}}$ becomes zero before reaching the minimum. The critical switching in the direct write mode occurs at $E_{\mathrm{M}}=0$, as was mentioned previously. The values of $E_{\mathrm{M}}$ at intermediate $H$ values and also near the peak differ substantially depending on $\Delta t$, yet these different values converge into a single value as $H$ approaches $\sim 1500$ Oe. This convergence indicates that all the anisotropies (and hence the shape anisotropy) are identical, since they are independent of $\Delta t$ in this high field range. The behavior is due to the constructive coupling of the two magnetostatic fields, $H_{\text {dem }}$ and $H_{\text {dip }}$, as the magnetic configuration approaches saturation. Identical shape anisotropy indicates the identical sum of $H_{\mathrm{dem}}$ and $H_{\mathrm{dip}}$ in both the $x$ and $y$ directions, although the respective values of $H_{\mathrm{dem}}$ and $H_{\mathrm{dip}}$ differ. This indicates that a smaller self-demagnetizing field in the thinner layer for example, is exactly compensated by a larger dipole field from the alternate thicker layer, when the magnetizations in the two magnetic layers are parallel (namely, saturation occurs). It must be remembered that the total thickness of the two magnetic layers is fixed at $4 \mathrm{~nm}$ in this study.

\section{Conclusions}

Thermal stability of nanostructured synthetic ferrimagnets has been examined in this study as a function of $H$ by using an accurate analytical expression for $E$. A simple single domain model is used in the derivation of the energy equation, yet the energies due to magnetostatic interactions are accurately taken into account. 3D FEM is employed to calculate the effective values of $H_{\mathrm{dem}}$ and $H_{\text {dip }}$ by averaging the non-uniform fields over the entire magnetic layer and these effective fields are used as inputs in an analytical equation for $E$. Elliptical cells are considered that have lateral dimensions of $160 \mathrm{~nm}$ (long axis) $\times 80 \mathrm{~nm}$ (short axis) and varying values of $\Delta t$. The direction of $H$, which points to the $45^{\circ}$ direction, is in the opposite direction to the thicker layer magnetization. At the remanent state, the value of $E_{\mathrm{M}}$, which is a direct measure of the thermal stability, increases with the increase of $\Delta t$, but its order is reversed within a very small field range $(<55 \mathrm{Oe})$ because the decrease of the magnetic energy barrier is steeper at larger $\Delta t$ due to stronger Zeeman effects. At small thickness asymmetries of $\Delta t=$ 0.2 and $0.4 \mathrm{~nm}$, a minimum occurs at $H=350 \sim 450 \mathrm{Oe}$, while no minimum is observed at large thickness asymmetries of $\Delta t \geq 0.6 \mathrm{~nm}$ because the value of $E_{\mathrm{M}}$ becomes zero, where the magnetization switching occurs in the direct write mode, before reaching the minimum. The values of $E_{\mathrm{M}}$ at intermediate $H$ values and also near the peak differ substantially depending on $\Delta t$, but these different values converge into a single value as $H$ approaches $\sim 1500$ Oe, indicating that the shape anisotropies are identical since they are independent of $\Delta t$. The behavior is due to the constructive coupling of the two magnetostatic fields, $H_{\mathrm{dem}}$ and $H_{\mathrm{dip}}$, as the magnetic configuration approaches saturation. Specifically, the sum of $H_{\text {dem }}$ and $H_{\text {dip }}$ in both the $x$ and $y$ directions is nearly the same for all the $\Delta t$ values, although the respective values of $H_{\text {dem }}$ and $H_{\text {dip }}$ differ. This indicates that a smaller selfdemagnetizing field in the thinner layer for example, is exactly compensated by a larger dipole field from the alternate thicker layer. A significant difference was found in the results calculated by using the simplifying assumption, whereby the self-demagnetizing field is identical in magnitude to the dipole field. At a small thickness asymmetry of $0.2 \mathrm{~nm}$ for example, the value of $E_{\mathrm{M}}$ is reduced from $68 \mathrm{kT}(T=300 \mathrm{~K})$ to $6 \mathrm{kT}$ at the remanent state and a progressive switching behavior changes into a critical direct writing switching mode, as the simplifying assumption is used. The present results clearly emphasize the importance of using an accurate analytical equation for $E$ in predicting the thermal stability of nanostructured synthetic ferrimagnets.

\section{Acknowledgments}

This work was supported by the KIST-KU DRC for Spintronics.

\section{References}

[1] D. C. Worledge, Appl. Phys. Lett. 84, 4559 (2004).

[2] J. K. Han, K. H. Shin, and S. H. Lim, J. Magn. Magn. Mater. 310, 2339 (2007). 
[3] J. K. Han, K. H. Shin, and S. H. Lim, J. Appl. Phys. 101, 09F506 (2007).

[4] J. K. Han, J. H. NamKoong, and S. H. Lim, J. Phys. D 41, 232005 (2008).

[5] J. K. Han, C. W. Han, and S. H. Lim, J. Kor. Phys. Soc. 55, 2505 (2009).
[6] J. Hayakawa, S. Ikeda, Y. M. Lee, R. Sasaki, T. Meguro, F. Matsukura, H. Takahashi, and H. Ohno, Jpn. J. Appl. Phys. 45, L1057 (2006).

[7] D. C. Worledge, Appl. Phys. Lett. 84, 2847 (2004).

[8] C. W. Han, J. K. Han, and S. H. Lim, J. Appl. Phys. 106, 094508 (2009). 